\title{
L-series and Hurwitz zeta functions associated with the universal formal group
}

\author{
Piergiulio Tempesta
}

\begin{abstract}
The properties of the universal Bernoulli polynomials are illustrated and a new class of related L-functions is constructed. A generalization of the Riemann-Hurwitz zeta function is also proposed.

Mathematics Subject Classification (2010): 11M41 (primary); 55N22 (secondary).
\end{abstract}

\section{Introduction}

The aim of this article is to establish a connection between the theory of formal groups on one side and a class of generalized Bernoulli polynomials and Dirichlet series on the other side. Some of the results of this paper were announced in the communication [26].

We will prove that the correspondence between the Bernoulli polynomials and the Riemann zeta function can be extended to a larger class of polynomials, by introducing the universal Bernoulli polynomials and the associated Dirichlet series. Also, in the same spirit, generalized Hurwitz zeta functions are defined.

Let $R$ be a commutative ring with identity, and $R\left\{x_{1}, x_{2}, \ldots\right\}$ be the ring of formal power series in $x_{1}, x_{2}, \ldots$ with coefficients in $R$. We recall that a commutative one-dimensional formal group law over $R$ is a formal power series $\Phi(x, y) \in$ $R\{x, y\}$ such that

$$
\begin{aligned}
\Phi(x, 0) & =\Phi(0, x)=x \\
\Phi(\Phi(x, y), z) & =\Phi(x, \Phi(y, z)) .
\end{aligned}
$$

When $\Phi(x, y)=\Phi(y, x)$, the formal group law is said to be commutative. The existence of an inverse formal series $\varphi(x) \in R\{x\}$ such that $\Phi(x, \varphi(x))=0$ follows from the previous definition.

As is well known, formal groups are relevant in many branches of mathematics, especially in the theory of elliptic curves [25], in algebraic topology [5, 21], in analytic number theory [15] and in combinatorics [3].

This research has been supported by the Ministerio de Ciencia e Innovación, Spain, research project FIS2008-00200.

Received June 30, 2008; accepted in revised form March 22, 2009. 
Bernoulli polynomials are useful in many contexts, as in the theory of distributions in $p$-adic analysis, in the theory of modular forms, in the study of polynomial expansions of analytic functions, in interpolation theory, etc. Consequently, they have been generalized in many ways. Leopold and Iwasawa proposed a generalization, motivated by the theory of $p$-adic L-functions [18]. Several new applications in mathematical physics, in connection with the theory of the Korteweg-de Vries equation [13] and in the study of vertex algebras [11], also appeared recently.

The Bernoulli numbers are relevant in number theory, e.g. to compute rational values of the Riemann zeta function [8], in the theory of cyclotomic fields and, since Kummer's work, in connection with Fermat's last Theorem [17]. Standard applications in algebraic topology are found in the computation of Todd characteristic classes and in the Hirzebruch signature theorem. In the last years the theory of Bernoulli number identities has been connected with Quantum Field Theory [12] and has been useful in the computation of Gromov-Witten invariants [14].

In this paper, we will study a generalization of the Bernoulli polynomials, that we call the Universal Bernoulli polynomials (see Definition 2.1). They are indeed related to the Lazard universal formal group. The corresponding numbers by construction coincide with the universal Bernoulli numbers, introduced in [9]. They turn out to have an important role in complex cobordism theory (see e.g. [3], and [23]), where the coefficients $c_{n}$ are identified with the cobordism classes of $\mathbb{C} P^{n}$. They also obey generalizations of the celebrated Kummer and Clausen-von Staudt congruences [1]. In [19], they have been used to generate classes of hyperfunctions via suitable generalizations of the Lipschitz summation formula.

As noticed in [5], "all fundamental facts of the theory of unitary cobordisms, both modern and classical, can be expressed by means of Lazard's formal group". This observation motivates the present construction, which associates polynomial structures of Appell type, i.e. the universal Bernoulli polynomials to the Lazard formal group.

The main result of this work is the following: a new class of L-functions will be attached to the universal Bernoulli numbers, exactly in the same was as the Riemann zeta function is connected to the standard ones. A family of RiemannHurwitz series is also defined, in such a way that it corresponds to the generalized Bernoulli polynomials we propose is the same as the Hurwitz zeta function is related to the Bernoulli polynomials. We also clarify the relation between the formal group structure used in this paper and the finite operator calculus, as has been formulated by G. C. Rota [24].

The future research plans include the study of higher-order polynomial structures (case $a \neq 1$ ) and p-adic extensions of the theory of L- series and Hurwitz zeta functions analyzed in this paper. Also, the case of multidimensional polynomials will be considered.

ACKnowledgements. I am grateful to professors B. Dubrovin, R. A. Leo, S. Marmi, D. Zagier and U. Zannier for many useful discussions. I also wish to thank for kind hospitality the Centro di Ricerca Matematica Ennio De Giorgi, Scuola Normale Superiore, Pisa, where part of this work has been carried out. 


\section{Universal Bernoulli polynomials and numbers}

\subsection{First algebraic properties}

We recall the definition of universal Bernoulli polynomials, introduced in [26].

Definition 2.1. Let us consider the formal group logarithm, over the polynomial $\operatorname{ring} \mathbb{Q}\left[c_{1}, c_{2}, \ldots\right]$

$$
F(s)=s+c_{1} \frac{s^{2}}{2}+c_{2} \frac{s^{3}}{3}+\ldots
$$

Let $G(t)$ be the associated formal group exponential:

$$
G(t)=t-c_{1} \frac{t^{2}}{2}+\left(3 c_{1}^{2}-2 c_{2}\right) \frac{t^{3}}{6}+\ldots
$$

so that $F(G(t))=t$.

The universal higher-order Bernoulli polynomials $B_{k, a}^{G}\left(x, c_{1}, \ldots, c_{n}\right) \equiv B_{k, a}^{G}(x)$ are defined by

$$
\left(\frac{t}{G(t)}\right)^{a} e^{x t}=\sum_{k \geq 0} B_{k, a}^{G}(x) \frac{t^{k}}{k !}, \quad x, a \in \mathbb{R} .
$$

When $a=1, c_{i}=(-1)^{i}$, then $F(s)=\log (1+s), G(t)=e^{t}-1$, and the universal Bernoulli polynomials and numbers reduce to the standard ones. For sake of simplicity, we will put $B_{k, 1}^{G}(x) \equiv B_{k}^{G}(x)$ and $B_{k}^{G}(0) \equiv B_{k}^{G}$.

The numbers $B_{k, 1}^{G}(0) \in \mathbb{Q}\left[c_{1}, c_{2}, \ldots\right]$ coincide with Clarke's universal Bernoulli numbers [9]. The power series $\Phi\left(s_{1}, s_{2}\right)=G\left(F\left(s_{1}\right)+F\left(s_{2}\right)\right)$ defines the Lazard universal formal group [15]. It is defined over the Lazard ring $L$, i.e. the subring of $\mathbb{Q}\left[c_{1}, c_{2}, \ldots\right]$ generated by the coefficients of the power series $G\left(F\left(s_{1}\right)+F\left(s_{2}\right)\right)$. In cobordism theory, the coefficients $c_{n}$ are identified with the cobordism classes of $\mathbb{C} P^{n}$

The universal Bernoulli polynomials (2.3) possess many remarkable properties. By construction, for any choice of the sequence $\left\{c_{n}\right\}_{n \in \mathbb{N}}$ they represent a class of Appell polynomials. In the paper ([26]), several examples of Bernoulli-type polynomials of first and second kind, as well as related Euler polynomial sequences are studied.

We easily deduce the following results. The Appell property is expressed by the identity:

$$
B_{n, a}^{G}(x+y)=\sum_{k=0}^{n}\left(\begin{array}{l}
n \\
k
\end{array}\right) B_{k, a}^{G}(x) y^{n-k} .
$$

In particular, for $x=0$ we obtain a useful characterization of $B_{n, a}^{G}(x)$. 
Lemma 2.2. The higher-order universal Bernoulli polynomials are expressed in terms of the higher-order universal Bernoulli numbers by the relation

$$
B_{n, a}^{G}(x)=\sum_{k=0}^{n}\left(\begin{array}{l}
n \\
k
\end{array}\right) B_{k, a}^{G}(0) x^{n-k} .
$$

From relation (2.4), we get

$$
B_{n, a+b}^{G}(x+y)=\sum_{k=0}^{n}\left(\begin{array}{l}
n \\
k
\end{array}\right) B_{k, a}^{G}(x) B_{n-k, b}^{G}(y) .
$$

and for $(a+b)=0$

$$
(x+y)^{n}=\sum_{k=0}^{n}\left(\begin{array}{l}
n \\
k
\end{array}\right) B_{k, a}^{G}(x) B_{n-k,-a}^{G}(y) .
$$

In [27], it is shown that interesting realizations of the class of polynomials (2.3) can also be constructed using the finite operator theory, introduced by G. C. Rota [24]. Indeed, the formal exponential $G(t)$ is chosen to be the representative of a difference delta operator, as will be explained in Section 3.

\subsection{Periodic structures}

In several applications, it is useful to consider periodic polynomial structures. Here we consider a periodic version of our polynomials.

Definition 2.3. The periodic (higher-order) generalized Bernoulli polynomials related to the formal group $\mathrm{G}$ are defined by

$$
\overline{B_{n, a}^{G}}(x)=B_{n, a}^{G}(x-[x]),
$$

where $[x]$ is the integer part of $\mathrm{x}$.

Clearly, these polynomials coincide with those in Definition 2.1 in the fundamental domain $\left[0,1\left[\right.\right.$. It is easy to observe that the functions $\overline{B_{1, a}^{G}}(x)$ admit discontinuities at the integral values of $x$. Using the Appell property we immediately obtain, for $k \leq n-2$,

$$
{\overline{B_{n, a}^{G}}}^{(k)}(x)=n(n-1) \ldots(n-k+1) \overline{B_{n-k, a}^{G}}(x) .
$$

The derivative of order $n-1$ has a jump for every integral value of $x$. Finally, by deriving again we get

$$
\frac{d^{n}}{d x^{n}} \overline{B_{n, a}^{G}}(x)=n !\left(1-\sum_{k \in \mathbb{Z}} \delta(x-k)\right),
$$

where $\delta$ denotes the Dirac delta distribution. In $[19,20]$, these periodic structures have been connected with the theory of hyperfunctions in one variable. Indeed, the coefficients of the Fourier expansion of these polynomials can be used to define generalized polylogarithms and Lipschitz-type summation formulae. 


\subsection{Congruences}

The values $B_{n, 1}^{G}(0):=\widehat{B_{n}}$ correspond to the well-known universal Bernoulli numbers. Here we mention only two of their most relevant properties.

(i) The universal Von Staudt's congruence [9].

If $n$ is even,

$$
\widehat{B_{n}} \equiv-\sum_{\substack{p-1 \mid n \\ p \text { prime }}} \frac{c_{p-1}^{n /(p-1)}}{p} \quad \bmod \quad \mathbb{Z}\left[c_{1}, c_{2}, \ldots\right]
$$

If $n$ is odd and greater than 1 ,

$$
\widehat{B_{n}} \equiv \frac{c_{1}^{n}+c_{1}^{n-3} c_{3}}{2} \quad \bmod \quad \mathbb{Z}\left[c_{1}, c_{2}, \ldots\right] .
$$

When $c_{n}=(-1)^{n}$, the celebrated Clausen-Von Staudt congruence for Bernoulli numbers is obtained.

(ii) The universal Kummer congruences [1]. The numerators of the classical Bernoulli numbers play a special role, due to the Kummer congruences and to the notion of regular prime numbers, introduced in connection with the Last Fermat Theorem (see, for instance, [17]). The relevance of Kummer's congruences in algebraic geometry has been enlightened in [4]. More general versions of these congruences for the classical Bernoulli numbers are known in the literature [28]. As shown by Adelberg, the numbers $\widehat{B}_{n}$ satisfy a universal congruence. Suppose that $n \neq 0,1(\bmod p-1)$. Then

$$
\frac{\widehat{B}_{n+p-1}}{n+p-1} \equiv \frac{\widehat{B}_{n}}{n} c_{p-1} \quad \bmod \quad p \mathbb{Z}_{p}\left[c_{1}, c_{2}, \ldots\right]
$$

\section{Umbral calculus and formal groups}

There is a close connection between formal group exponentials and the finite operator theory. We recall some basic definitions.

Definition 3.1. The shift operator, denoted by $T$, is the operator whose action on a function is $T f(x)=f(x+\sigma)$, with $\sigma \in \mathbb{R}$. An operator $S$ commuting with $T$ is said to be shift-invariant.

Definition 3.2. A delta operator $Q$ is a shift-invariant operator such that $Q x=$ const $\neq 0$. 
As has been proved in [24], there is an isomorphism between the ring of formal power series in a variable $t$ and the ring of shift-invariant operators, carrying

$$
f(t)=\sum_{k=0}^{\infty} \frac{a_{k} t^{k}}{k !} \rightarrow \sum_{k=0}^{\infty} \frac{a_{k} Q^{k}}{k !} .
$$

Every delta operator has a unique sequence of associated basic polynomials, i.e. polynomials satisfying the following conditions:

$$
\begin{aligned}
& \text { 1) } \quad p_{0}(x)=1 ; \\
& \text { 2) } \quad p_{n}(0)=0 \text { for all } n>0 ; \\
& \text { 3) }
\end{aligned}
$$

Any shift invariant operator $S$ can also be expanded into a formal power series in terms of a delta operator $Q$. By using the isomorphism (3.1), a formal power series $s(t)$ is defined, which is called the indicator of $S$.

The main point of the correspondence between the two theories is that a shift invariant operator is a delta operator if and only if it corresponds, under the isomorphism (3.1), to a formal power series $g(t)$ such that $g(0)=0$ and $g^{\prime}(0) \neq 0$. This series admits a unique compositional inverse.

In this paper we are interested in realizations of delta operators in terms of finite difference operators. Therefore $G(t)$ is chosen to be a Laurent polynomial in $e^{t}$ (that we choose to have rational coefficients),

$$
G(t)=\sum_{k=l}^{m} a_{k} e^{k t}, \quad l, m \in \mathbb{Z}, l<m
$$

obeying two more constraints:

$$
\sum_{k=l}^{m} a_{k}=0, \quad \sum_{k=l}^{q+r} k a_{k}=1 .
$$

with $a_{m} \neq 0, a_{l} \neq 0$.

\section{Dirichlet series and formal groups}

In this section, we will associate new L-series with the polynomials (2.3). The Riemann zeta function is the most elementary example of the construction we propose. Let $\Gamma(s)=\int_{0}^{\infty} e^{-t} t^{s-1} d t$ be the Euler $\Gamma$-function. As is well known, if $\operatorname{Re} s>1$, $\zeta(s)=\frac{1}{\Gamma(s)} \int_{0}^{\infty} \frac{1}{e^{t}-1} t^{s-1} d t$. More generally, by imposing a suitable analytic constraint on the class of formal groups, we can attach to them an L-function. Precisely the following result holds. 
Theorem 4.1. Let $G(t)$ be a formal group exponential of the form (2.2), such that $1 / G(t)$ is a $C^{\infty}$ function over $\mathbb{R}_{+}$, rapidly decreasing at infinity.

(i) The function

$$
L(G, s)=\frac{1}{\Gamma(s)} \int_{0}^{\infty} \frac{1}{G(t)} t^{s-1} d t,
$$

defined for $\operatorname{Re} s>1$ admits a holomorphic continuation to the whole $\mathbb{C}$ and, for every $n \in \mathbb{N}$, we have

$$
L(G,-n)=(-1)^{n} \frac{B_{n+1}^{G}}{n+1} .
$$

(ii) Assume that $G(t)$ is also of the form (3.2). For $\operatorname{Re} s>1$ the function $L(G, s)$ has a representation in terms of a Dirichlet series

$$
L_{G}=\sum_{n=1}^{\infty} \frac{a_{n}}{n^{s}},
$$

where the coefficients $a_{n}$ are obtained as the coefficients of the formal expansion

$$
\frac{1}{G(t)}=\sum_{n=1}^{\infty} a_{n} e^{-n t}
$$

Assuming that $G(t) \geq e^{t}-1$, the series for $L_{G}$ is absolutely and uniformly convergent for $\operatorname{Re} s>1$, and

$$
\left|\sum_{n=1}^{\infty} \frac{a_{n}}{n^{s}}\right| \leq \sum_{n=1}^{\infty} \frac{1}{n^{\operatorname{Re} s}} .
$$

Remark 4.2. The condition (3.2) is not necessary for the existence of the series (4.3), but it is relevant for the actual computation of the numbers $a_{n}$.

Proof. Point (i) is in fact an adaptation of a standard result. For sake of clarity, here we sketch a proof, closely following an argument of P. Colmez, in [7], Chapter II.

Let $g(t)=\frac{1}{G(t)}$ be a $C^{\infty}$ function over $\mathbb{R}_{+}$, rapidly decreasing at infinity, and $\gamma(t)$ be a $C^{\infty}$ function over $\mathbb{R}_{+}$, taking the value 1 on $[0,1]$ and 0 on $[2,+\infty[$. It follows that $g(t)=\gamma g+(1-\gamma) g$ and $L(g, s)=L(\gamma g, s)+L((1-\gamma) g, s)$. The integral

$$
\int_{0}^{+\infty} g(t) t^{s} \frac{d t}{t}
$$

defines a holomorphic function on $C$, since $(1-\gamma) g$ vanishes in a neighborhood of 0 and rapidly decreases at infinity. We can always assume that $g$ has compact support, up to the replacement $g \rightarrow(1-\gamma) g$ since $L((1-\gamma) g,-n)=0 \forall n \in N$. 
An integration by parts show that the functional relation $L(G, s)=-L\left(G^{\prime}, s+1\right)$ holds for $\operatorname{Re} s>1$, allowing a holomorphic continuation of $g$. Now, we also have

$$
L(g,-n)=(-1)^{n+1} L\left(g^{(n+1)}, 1\right)=(-1)^{n+1} g^{(n)}(0)
$$

(ii) Observe that

$$
\begin{aligned}
\left|\sum_{n=1}^{\infty} \frac{a_{n}}{n^{s}}\right| & =\left|\frac{1}{\Gamma(s)} \int_{0}^{\infty} \frac{1}{G(t)} t^{s-1} d t\right| \leq\left|\frac{1}{\Gamma(s)}\right|\left|\int_{0}^{\infty} \frac{1}{G(t)} t^{s-1} d t\right| \\
& \leq\left|\frac{1}{\Gamma(s)}\right|\left|\int_{0}^{\infty} \frac{1}{e^{t}-1} t^{s-1} d t\right| \\
& =\left|\frac{1}{\Gamma(s)}\right|\left|\sum_{n=1}^{\infty} \frac{1}{n^{s}}\right| \leq \sum_{n=1}^{\infty} \frac{1}{n^{\operatorname{Res}}} .
\end{aligned}
$$

A simple consequence of the previous theorem is that the coefficients of the series $L_{G}$ satisfy the condition $a_{n}=O\left(n^{c}\right)$ for some $c>0$.

Remark 4.3. A construction of Dirichlet series based on formal group laws is known in the literature [15]. In ([16]), zeta functions of group varieties are related to formal group laws. One of the advantages of the construction proposed here is that the associated L-functions are related to the universal Bernoulli numbers, whose algebraic and combinatorial properties are particularly rich.

\section{Generalized Hurwitz zeta functions}

The previous ideas can be used to generalize the Hurwitz zeta function and its connection with the classical Bernoulli polynomials. The Hurwitz zeta function is defined by the series

$$
\zeta(s, a)=\sum_{n=0}^{\infty}(n+a)^{-s}
$$

for $a>0$. As is well known, this series converges absolutely for $\operatorname{Re} \mathrm{s}>1$ and it extends to a meromorphic function in $\mathbb{C}$ represented as a Mellin transform

$$
\zeta(s, a)=\frac{1}{\Gamma(s)} \int_{0}^{\infty} \frac{e^{-a x}}{1-e^{-x}} x^{s-1} d x=\frac{1}{\Gamma(s)} \int_{0}^{\infty} \frac{e^{x(1-a)}}{e^{x}-1} x^{s-1} d x
$$

with the obvious properties

$$
\zeta(s)=\zeta(s, 1)
$$


and

$$
\zeta(s, a+1)=\zeta(s, a)-a^{-s} .
$$

The Hurwitz zeta function takes the special values

$$
\zeta(-k, a)=-\frac{B_{k+1}(a)}{k+1},
$$

where $B_{k}(x)$ are the classical Bernoulli polynomials. It seems natural to propose the following generalization of $\zeta(s, a)$.

Definition 5.1. Let $G(t)$ be a formal group exponential, such that $1 / G(t)$ is a $C^{\infty}$ function over $\mathbb{R}_{+}$, rapidly decreasing at infinity. The generalized Hurwitz zeta function associated with $G$ is the function $\zeta^{G}(G, s, a)$, defined for $\operatorname{Re} s>1$ and $a>0$ by

$$
\zeta^{G}(s, a)=\frac{1}{\Gamma(s)} \int_{0}^{\infty} \frac{e^{-a x}}{G(x)} x^{s-1} d x .
$$

Assuming that $G(t)$ has the form (3.2), we can easily give to $\zeta^{G}(s, a)$ a formal series representation. Indeed

$$
\begin{aligned}
\zeta^{G}(s, a) & =\frac{1}{\Gamma(s)} \int_{0}^{\infty} \sum_{n=0}^{\infty} a_{n} e^{-n x} e^{-a x} x^{s-1} d x \\
& =\frac{1}{\Gamma(s)} \sum_{n=0}^{\infty} a_{n} \int_{0}^{\infty} e^{-n x} e^{-a x} x^{s-1} d x \\
& =\sum_{n=0}^{\infty} \frac{a_{n}}{(n+a)^{s}} .
\end{aligned}
$$

Corollary 5.2. The following property holds:

$$
\zeta^{G}(-k, a)=-\frac{B_{k+1}^{G}(a)}{k+1},
$$

where $B_{k}^{G}(x)$ is the $k$-th Bernoulli-type polynomial associated to the considered formal group.

Introduce the notation

$$
L(G, a)=\sum_{n=0}^{\infty} \frac{a_{n}}{(n+a)^{s}} .
$$

Again, if we restrict to the case (3.2), and assuming $G(x) \geq e^{x}-1$, the series $L(G, a)$ is absolutely convergent for $\operatorname{Re} s>1$, and

$$
\left|\sum_{n=1}^{\infty} \frac{a_{n}}{(n+a)^{s}}\right| \leq \sum_{n=1}^{\infty} \frac{1}{(n+a)^{\operatorname{Re} s}} .
$$


Observe that

$$
\frac{\partial}{\partial a} \zeta^{G}(s, a)=-s \zeta^{G}(s+1, a)
$$

and

$$
\zeta^{G}(s, x+y)=\sum_{k=0}^{\infty} \frac{y^{k}}{k !} \frac{\partial^{k}}{\partial x^{k}} \zeta^{G}(s, x)=\sum_{k=0}^{\infty}\left(\begin{array}{c}
s+k-1 \\
s-1
\end{array}\right)\left(-y^{k}\right) \zeta^{G}(s+k, x) .
$$

Let us consider the Dirichlet L-series of the form

$$
L(G, \theta, s)=\sum_{n=1}^{\infty} \frac{\theta(n) a_{n}}{n^{s}}
$$

where $\{\theta(n)\}$ is a sequence of numbers, periodic of period $f$ :

$$
\theta(n+f)=\theta(n), \quad \forall n \in \mathbb{Z} .
$$

The structures (5.6) can be easily related to the generalized Hurwitz function:

$$
\begin{aligned}
\sum_{n=1}^{\infty} \frac{\theta(n) a_{n}}{n^{s}} & =\sum_{k=1}^{f} \sum_{m=0}^{\infty} \theta(k+m f)(k+m f)^{-s} a_{k+m f} \\
& =\sum_{k=1}^{f} \theta(k) \sum_{m=0}^{\infty}(k+m f)^{-s} a_{k+m f} \\
& =\sum_{k=1}^{f} \theta(k) f^{-s} \sum_{m=0}^{\infty} a_{k+m f}\left(m+\frac{k}{f}\right)^{-s},
\end{aligned}
$$

so that we infer

$$
L(G, \theta, s)=f^{-s} \sum_{k=1}^{f} \theta(k) \zeta^{G}\left(s, \frac{k}{f}\right) .
$$

Consequently, we get

$$
L(G, \theta,-m)=-\frac{f^{m}}{m+1} \sum_{k=1}^{f} \theta(k) B_{m+1}^{G}\left(\frac{a}{f}\right) .
$$

More relevant is the case when we deal with Dirichlet characters. We have the following definition.

Definition 5.3. Let $\chi$ be a nontrivial Dirichlet character of conductor $f$. The universal Bernoulli $\chi$-numbers $B_{n, \chi}^{G}$ associated with the formal group exponential $G$ are defined by

$$
B_{n, \chi}^{G}:=f^{n-1} \sum_{a=1}^{f} \chi(a) B_{n}^{G}\left(\frac{a}{f}\right)
$$


Taking into account the summation formula for the universal Bernoulli polynomials, i.e.

$$
B_{n}^{G}(x)=\sum_{k=0}^{n}\left(\begin{array}{l}
n \\
k
\end{array}\right) B_{n-k}^{G} x^{k},
$$

valid since they represent an Appell sequence for any choice of $G$, we obtain another equivalent representation for the numbers $B_{n, \chi}^{G}$ :

$$
B_{n, \chi}^{G}=\sum_{a=1}^{f} \chi(a) \sum_{k=0}^{n}\left(\begin{array}{l}
n \\
k
\end{array}\right) B_{k}^{G} a^{n-k} f^{k-1} .
$$

The connection between our generalized Hurwitz functions and the corresponding zeta functions is straightforward:

$$
L(G, \chi, s)=\frac{1}{f^{s}} \sum_{n=1}^{f} \chi(n) \zeta^{G}\left(s, \frac{n}{f}\right)
$$

\section{References}

[1] A. Adelberg, Universal higher order Bernoulli numbers and Kummer and related congruences, J. Number Theory 84 (2000), 119-135.

[2] G. Almkvist and A. Meurman, Values of Bernoulli polynomials and Hurwitz's zeta function at rational points, C. R. Math. Acad. Sci. Soc. R. Can. 13 (1991), 104-108.

[3] A. BAKER, "Combinatorial and Arithmetic Identities Based on Formal Group Laws", Lecture Notes in Math., Vol. 1298, Springer, 1987, 17-34.

[4] A. Baker, F. Clarke, N. Ray and L. Schwartz, On the Kummer congruences and the stable homotopy of BU, Trans. Amer. Math. Soc. 316 (1989), 385-432.

[5] V. M. BukhshtABer, A. S. MishChenko and S. P. Novikov, Formal groups and their role in the apparatus of algebraic topology, Uspehi Mat. Nauk 26 (1971), 161-154.

[6] K. BARTZ and J. RUTKOwSKI, On the von Staudt-Clausen theorem, C. R. Math. Acad. Sci. Soc. R. Can. 15 (1993), 46-48.

[7] N. BERLINE and C. SABBAH (ets.), "La fonction zêta", Éditions de l'École polytechnique, 2003.

[8] D. CViJoviC and J. KLINOwsKi, New formulae for the Bernoulli and Euler polynomials at rational arguments, Proc. Amer. Math. Soc. 123 (1995), 1527-1535.

[9] F. Clarke, The universal Von Staudt theorems, Trans. Amer. Math. Soc. 315 (1989), 591603.

[10] L. E. Dickson, "History of the Theory of Numbers", Chelsea Publishing Company, 1971.

[11] B. Doyon, J. LEPOWSKY and A. MILAS, Twisted vertex operators and Bernoulli polynomials, arXiv: math. QA/0311151 (2003).

[12] G. V. DUNNE and C. SCHUBERT, Bernoulli number identities from quantum field theory, arXiv: math. NT/0406610 (2004).

[13] D. B. FAIRLIE and A. P. Veselov, Faulhaber and Bernoulli polynomials and solitons, Physica D 152-153 (2001), 47-50.

[14] C. FABER and R. PANDhARIPANDE, Hodge integrals and Gromov-Witten theory, Invent. Math. 139 (2000), 173-199. 
[15] M. Hazewinkel, "Formal Groups and Applications", Academic Press, New York, 1978.

[16] T. HondA, Formal groups and zeta-functions, Osaka J. Math. 5 (1968), 199-213.

[17] K. IREland and M. Rosen, "A Classical Introduction to Modern Number Theory", Springer-Verlag, 1982.

[18] K. IwASAWA, "Lectures on $p$-adic L-Functions", Princeton University Press, Princeton, 1972.

[19] S. MARmi and P. Tempesta, Polylogarithms, hyperfunctions and generalized Lipschitz summation formulae, Preprint Scuola Normale Superiore, Centro di Ricerca Matematica "Ennio De Giorgi" 1-2007, arXiv: 0712.1046v1 [math/NT] (2007).

[20] S. MARMI and P. TEMPESTA, On the relation between formal groups, In: "Appell Polynomials and Hyperfunctions, Symmetry and Perturbation Theory", G. Gaeta, R. Vitolo and S. Walcher (eds.), World Scientific, 2007, 132-139.

[21] S. P. Novikov, The methods of algebraic topology from the point of view of cobordism theory, Izv. Akad. Nauk SSSR Ser. Mat. 31 (1967), 885-951, transl. Math. SSR-Izv. 1 (1967), 827-913.

[22] A. OGg, "Modular Forms and Dirichlet Series", Mathematics Lecture Note Series, W. A. Benjamin, Inc., 1969.

[23] N. RAY, Stirling and Bernoulli numbers for complex oriented homology theory, In: "Algebraic Topology", G. Carlsson, R. L. Cohen, H. R. Miller and D. C. Ravenel (eds.), Lecture Notes in Math., Vol. 1370, Springer-Verlag, 1986, 362-373,

[24] G. C. RotA, "Finite Operator Calculus", Academic Press, New York, 1975.

[25] J-P. SERRE, Courbes elliptiques et groupes formels, Annuaire du Collège de France (1966), 49-58. (Oeuvres, Vol. II, 71, 315-324.)

[26] P. Tempesta, Formal Groups, Bernoulli-type polynomials and L-series, C. R. Math. Acad. Sci. Paris, Ser. I 345 (2007), 303-306.

[27] P. Tempesta, On new Appell sequences of polynomials of Bernoulli and Euler type, J. Math. Anal. Appl. 341 (2008), 1295-1310.

[28] P. T. Young, Congruence for Bernoulli, Euler and Stirling Numbers, J. Number Theory 78 (1999), 204-227.

Departamento de Fisica Teorica II

Universidad Complutense

Ciudad Universitaria

28040 Madrid, Spain

p.tempesta@fis.ucm.es 